\title{
Deficiency of X-Linked Protein Kinase Nrk during Pregnancy Triggers Breast Tumor in Mice
}

\author{
Takayo Yanagawa, ${ }^{* \dagger}$ Kimitoshi Denda, ${ }^{*}$ Takuya Inatani, ${ }^{*}$ Toshiaki Fukushima, ${ }^{*}$ Toshiaki Tanaka, ${ }^{*}$ Nobue Kumaki, \\ Yutaka Inagaki, ${ }^{\dagger \ddagger}$ and Masayuki Komada ${ }^{\star \S}$
}

\begin{abstract}
From the Department of Biological Sciences* and the Cell Biology Unit,$^{\S}$ Institute of Innovative Research, Tokyo Institute of Technology, Yokohama; the Center for Matrix Biology and Medicine, ${ }^{\dagger}$ Graduate School of Medicine, Tokai University, Isehara; and the Departments of Regenerative Medicine ${ }^{\ddagger}$ and Pathology, ${ }^{\circledR}$ Tokai University School of Medicine, Isehara, Japan
\end{abstract}

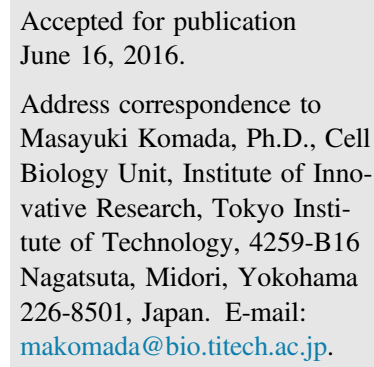

The onset and/or growth of breast tumor are controlled, at least in part, by estrogen. Therefore, to prevent the development of breast tumor, estrogen-dependent proliferation of mammary epithelial cells during pregnancy needs to be suppressed once the mammary gland is fully developed to enable lactation. However, the underlying molecular mechanisms remain unknown. Nrk is an X-linked protein serine/threonine kinase in the germinal center kinase family. Herein, we demonstrate a frequent occurrence of breast tumors in homozygous and heterozygous Nrk mutant mice that have experienced pregnancy/parturition. The tumors never developed in the mutant mice without a history of pregnancy/ parturition. They exhibited histopathological features of noninvasive tubular adenocarcinoma, and expressed estrogen receptor $\alpha$. At late gestation when estrogen receptor $\alpha$ expression was significantly reduced in the wild-type mammary gland, grossly normal mammary glands in the pregnant Nrk mutant mice occasionally contained hyperplastic foci continuously expressing the receptor. Consistently, Nrk expression was induced in the wild-type mammary gland at this period of pregnancy. On the other hand, the pregnant Nrk mutant mice also showed elevated blood estrogen levels at late gestation. We suggest that Nrk suppresses the excessive proliferation of mammary epithelial cells during pregnancy, and the impairment of this regulatory system leads to frequent occurrence of breast tumor in Nrk mutant mice. (Am J Pathol 2016, 186: 2751-2760; http://dx.doi.org/10.1016/j.ajpath.2016.06.005)
The prevalence of breast cancer is increasing worldwide. The development of breast cancer depends on many risk factors, such as genetic susceptibility ${ }^{1,2}$ and postmenopausal obesity. ${ }^{3,4}$ In the latter case, increased local estrogen production in the breast adipose tissue has been implicated in cancer development in postmenopausal women. ${ }^{5,6}$ Indeed, one of the most characteristic features of breast cancer is its estrogen dependency, ${ }^{7}$ and a growing body of clinical evidence indicates that estrogen plays a critical role in breast cancer development. Breast cancer is known to develop preferentially in women who have experienced a higher number of menstrual cycles owing to early menarche and/or late menopause and therefore had a greater chance of exposure to estrogen. ${ }^{8}$ Moreover, it has been debated whether the risk of breast cancer is increased in women who are administered oral contraceptives $^{9}$ or postmenopausal hormone replacement therapy. ${ }^{10}$ In concordance with these epidemiological data, estrogen receptor (ER) is frequently expressed in adenoma and low-grade carcinoma cells of the mammary gland origin. The ER-positive breast tumor cells hyperproliferate in response to this hormone, and anti-estrogen treatment is effective in patients with ER-positive breast cancer. ${ }^{11}$ For instance, the estrogen antagonist, tamoxifen, is useful in preventing the recurrence of breast cancer. ${ }^{12}$ Inhibitors for CYP19A1 (P450 aromatase), a key enzyme in estrogen synthesis, are also in clinical use to inhibit the conversion of testosterone to estrogen in postmenopausal patients with breast cancer. ${ }^{13}$

In premenopausal women, in addition to a periodic increase of blood estrogen levels during the physiological menstrual

Supported by Japan Society for the Promotion of Science KAKENHI grants 15K14378 (K.D.) and 24650610 (M.K.) and a Tokai University School of Medicine Research Aid (T.Y.).

Disclosures: None declared. 
cycle, estrogen production is remarkably up-regulated during pregnancy; the blood estrogen levels at the third trimester increase $>10$-fold compared with the late follicular phase of the menstrual cycle in humans. ${ }^{14}$ The elevated levels of estrogen during pregnancy, together with progesterone, promote the physiological hyperplasia of the mammary gland to enable lactation by inducing the proliferation of mammary epithelial cells. ${ }^{15}$ Once the mammary gland is fully developed, the level of estrogen signaling must be down-regulated to prevent the excessive hyperplasia of the mammary gland that potentially leads to the development of breast tumor. However, the molecular and cellular mechanisms that suppress the excessive proliferation of mammary epithelial cells at late gestation remain poorly understood. Moreover, it is unknown whether dysregulated estrogen signaling during pregnancy leads to excessive mammary gland hyperplasia and subsequent development of breast cancer. ${ }^{16,17}$

Nrk is an X chromosome-linked protein serine/threonine kinase in the germinal center kinase family. ${ }^{18,19}$ In mice, Nrk is specifically expressed in the embryo ${ }^{18,19}$ and placenta. ${ }^{20}$ Although little is known about its molecular and cellular functions, ${ }^{21,22}$ we have previously shown that a disruption of the Nrk gene in mice causes the hyperproliferation of fetus-derived spongiotrophoblasts in placenta, resulting in placental overgrowth [greater than twofold in weight than wild-type (WT) placenta] and a severe defect in the induction of parturition. ${ }^{20}$ In the present study, we demonstrate another female-specific critical role for Nrk. We provide evidence that Nrk plays a role in suppressing the development of breast tumor associated with the mammary gland hyperplasia during pregnancy.

\section{Materials and Methods}

\section{Mice}

Mutant mice harboring a deletion of the first exon of the Nrk gene were generated as described previously. ${ }^{20}$ All mice used in the present study received humane care, and those carrying the breast tumor were euthanized before the diameter of the tumor reached approximately $3 \mathrm{~cm}$. All animal experiments were approved by and performed under the guidelines of the Institutional Animal Care Research Advisory Committee of Tokyo Institute of Technology. PCR genotyping of fetal and neonatal Nrk mutant mice was performed on yolk sac and tail biopsies, respectively, as previously described. ${ }^{20}$ PCR analysis of Sry, a sex-determining gene on the $\mathrm{Y}$ chromosome, was used to distinguish male and female fetuses/placentas, as previously described. ${ }^{20}$

\section{Histopathological Examination}

Mammary glands and breast tumors were dissected from mice, fixed in $10 \%$ neutral formaldehyde for 16 hours at room temperature, gradually dehydrated in ethanol, and embedded in paraffin. Paraffin sections ( $2 \mu \mathrm{m}$ thick) were cut, deparaffinized with xylene, and subjected to hematoxylin and eosin staining using the standard protocol. For immunohistochemical staining, the tissue sections were subjected to permeabilization in $0.2 \%$ Triton X-100 in Tris-buffered saline for 10 minutes, optimal antigen retrieval using $10 \mathrm{mmol} / \mathrm{L}$ citric acid buffer ( $\mathrm{pH}$ 6.0) for 20 minutes at $98^{\circ} \mathrm{C}$, and incubation with antibodies recognizing ER $\alpha(1 \mu \mathrm{g} / \mathrm{mL}$; Santa Cruz Biotechnology, Dallas, TX), Ki-67 (5 $\mu \mathrm{g} / \mathrm{mL}$; BioLegend, San Diego, CA), or ErbB2 (10 $\mu \mathrm{g} / \mathrm{mL}$; Abcam, Cambridge, UK) for 1 hour at room temperature. They were subsequently incubated with horseradish peroxidase-conjugated polymerized secondary antibodies recognizing rabbit or mouse IgG (Dako, Glostrup, Denmark), and visualized using diaminobenzidine (Dako). Proliferation of mammary glands was quantified by measuring the mammary epithelial cell areas using the cellSens software version 1.6 (Olympus, Tokyo, Japan) equipped in a light microscope BX63 (Olympus). The number of ER $\alpha$ - and Ki-67-positive cells was counted manually under the microscope and expressed as values against the whole cell number per unit area.

\section{RT-PCR and Real-Time RT-PCR}

Total RNA was isolated from the mammary gland, ovary, and placenta of mice using the ISOGEN RNA extraction kit (Nippon Gene, Tokyo, Japan), and reverse-transcribed using the SuperScript III First-Strand Synthesis System for RTPCR (Invitrogen, Carlsbad, CA). RT-PCR analysis of Nrk expression was performed as previously described. ${ }^{20}$ The amounts of Nrk, ER $\alpha$, and CYP19A1 mRNAs were quantified using the real-time RT-PCR analysis, as previously described. ${ }^{23} \mathrm{~A}$ set of gene-specific PCR primers and an oligonucleotide probe were selected for each mRNA according to the manufacturer's recommendation (Applied Biosystems, Foster City, CA). The mRNA levels were normalized against that of glyceraldehyde 3-phosphate dehydrogenase in each RNA preparation.

\section{DNA Constructs and Transfection}

For the construction of the luciferase reporter vector harboring the estrogen responsive element (ERE), oligonucleotides containing three copies of the ERE sequence (5'AGGTCACAGTGACCT $\left.-3^{\prime}\right)^{24}$ were chemically synthesized, annealed, and cloned into a luciferase reporter vector, pNL3.2 (Promega, Madison, WI). Expression vectors for the WT and kinase-dead (substitution of $\mathrm{Lys}^{54}$ to Glu, K54E) mutant of murine Nrk were constructed as described previously. ${ }^{18}$ DNA transfection was performed using polyethylenimine (Polysciences, Niles, IL).

\section{Cell Culture and Immunoblotting}

The MCF-7 human breast cancer cell line was obtained from the RIKEN BioResource Center (Tsukuba, Japan) through the National Bio-Resource Project of the Ministry 
of Education, Culture, Sports, Science and Technology, Japan, and maintained in Dulbecco's modified Eagle's medium supplemented with $10 \%$ fetal bovine serum and nonessential amino acids. For immunoblotting, cells were solubilized in boiling $62.5 \mathrm{mmol} / \mathrm{L}$ Tris- $\mathrm{HCl}, \mathrm{pH} 6.8,5 \%$ SDS, $10 \%$ glycerol, 5\% 2-mercaptoethanol, and 0.01\% bromophenol blue. After protein separation by SDS-PAGE and protein transfer to polyvinylidene difluoride membranes, the membranes were immunoblotted with anti-ER $\alpha$ antibody (1:1000; Thermo Fisher Scientific, Fremont, CA). Blots were detected using the ECL Western Blotting Detection Reagents (GE Health Care, Piscataway, NJ). To examine the phosphorylation state of ER $\alpha$, cell lysates were separated by SDS-PAGE containing Phos-tag (Wako Pure Chemical, Osaka, Japan) according to the manufacturer's instructions.

\section{Luciferase Reporter Assay}

Cells were grown in Dulbecco's modified Eagle's medium supplemented with $10 \%$ charcoal-treated fetal bovine serum/nonessential amino acids and depleted of phenol red in 96-well plates, and cotransfected with the ERE luciferase reporter vector $(15 \mathrm{ng})$ and the Nrk expression vector (70 ng). After 6 hours of incubation, cells were cultured in the presence or absence of $10 \mathrm{nmol} / \mathrm{L}$ estradiol (Wako Pure Chemical) for 48 hours. The luciferase activity in the cells was determined using the Nano-Glo dual-luciferase reporter assay system (Promega) according to the manufacturer's instructions. The pGL4.54 vector (Promega), harboring a thymidine kinase promoter-driven firefly luciferase gene, was used as an internal control.

\section{Measurement of Serum Estradiol and Progesterone Concentrations}

Blood was collected from the abdominal vena cava of pregnant mice at 17.5 days postcoitum $(\mathrm{dpc})$, coagulated, centrifuged at $2000 \times g$ for 20 minutes at $4^{\circ} \mathrm{C}$, and the supernatant (serum) was recovered. The serum concentrations of estradiol and progesterone were measured by liquid chromatography-tandem mass spectrometry at ASKA Pharma Medical (Kawasaki, Japan). ${ }^{25,26}$

\section{Statistical Analysis}

Group results were expressed as means \pm SD. Wilcoxon's test was used to evaluate the statistical significance between groups. The cumulative incidence of breast tumor was compared by the log-rank test. $P<0.05$ was considered to be statistically significant.

\section{Results}

\section{Frequent Occurrence of Breast Tumor in Nrk Mutant Mice after Experiencing Pregnancy/Parturition}

During the maintenance of the Nrk mutant mouse colony, we sometimes observed large $(>1 \mathrm{~cm}$ in diameter) breast tumors in homozygous $\left(\mathrm{Nrk}^{-/-}\right)$and heterozygous $\left(\mathrm{Nrk}^{+/-}\right)$ mutant female mice only after they experienced pregnancy/ parturition. The breast tumor never developed until the age of 2 years in Nrk mutant mice that had no history of pregnancy/parturition. To examine this phenotype in a quantitative manner, we compared the incidences of breast

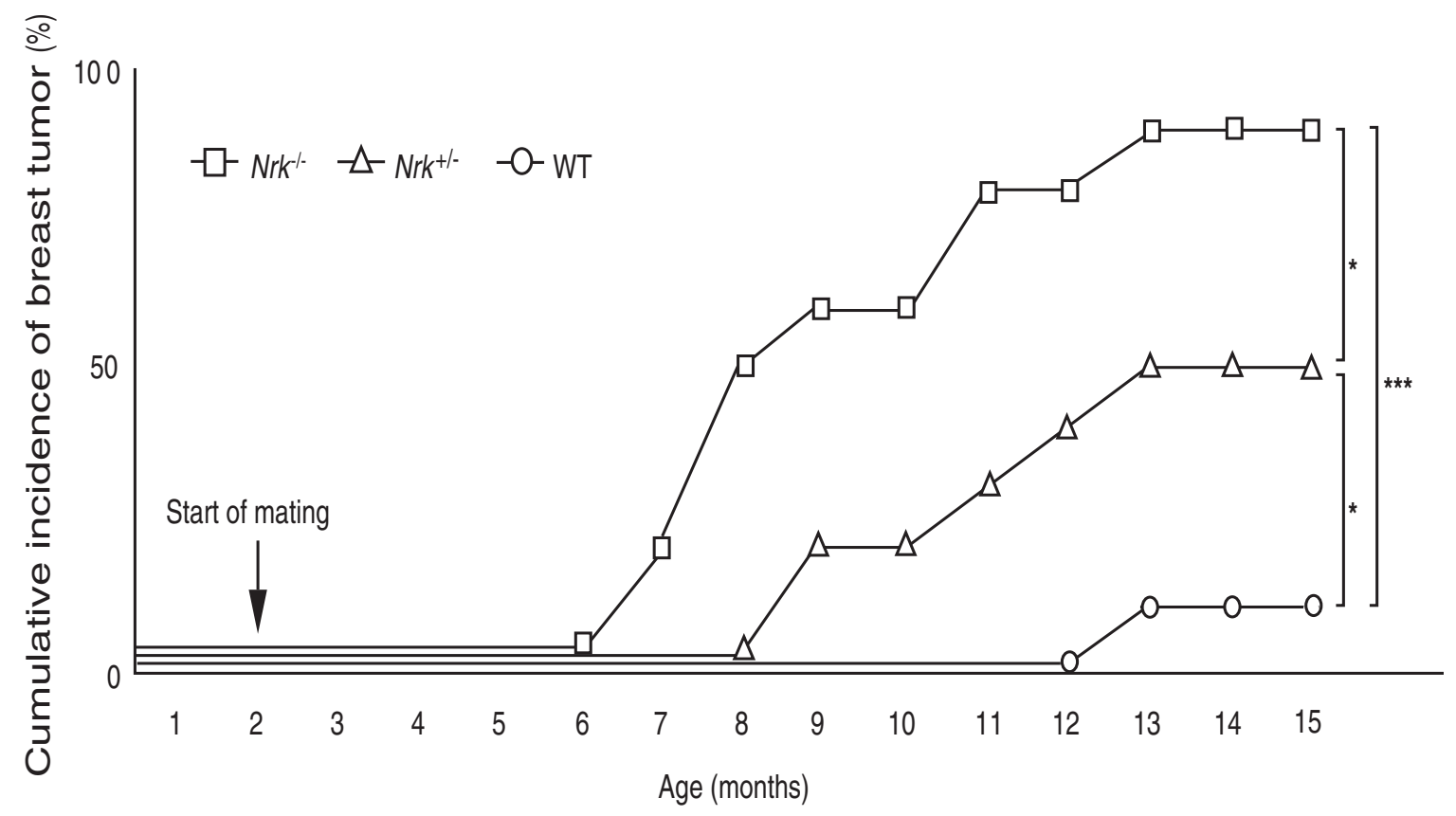

Figure 1 Cumulative incidence of breast tumor in Nrk mutant female mice that have experienced pregnancy/parturition. $\mathrm{Nrk}^{-/-}$, Nrk ${ }^{+/-}$, and wild-type (WT) female mice were continuously mated with WT male mice from 2 to 15 months of age. The cumulative incidences of breast tumor in the three groups of mice are shown. $n=10$ for each group. ${ }^{*} P<0.05,{ }^{* *} P<0.001$. 
tumor occurrence in $10 \mathrm{Nrk}^{-/-}, 10 \mathrm{Nrk}^{+/-}$, and $10 \mathrm{WT}$ female mice during continuous mating (from 2 to 15 months of age) with WT male mice. Apparent breast tumors were first observed in $\mathrm{Nrk}^{-/-}$and $\mathrm{Nrk}^{+--}$mutants at 7 to 9 months of age, and the number of mice harboring breast tumor increased as they grew older (Figure 1). By the age of 15 months, nine $\mathrm{Nrk}^{-l-}$ (90\%) and five $\mathrm{Nrk}^{+-}$(50\%) mice had breast tumors. The tumors developed in any of the six mammary glands in mice. In contrast, only one WT mice (10\%) exhibited a breast tumor at the age of 13 months (Figure 1). There was a significant difference among groups in the cumulative incidences of breast tumors $(P<0.05)$.

\section{Breast Tumors in Nrk Mutant Mice Exhibit Histopathological Features of Noninvasive Tubular Adenocarcinoma}

Breast tumors in the Nrk mutant mice grew rapidly in an outward manner (Figure 2A). Histopathological examination revealed that the tumor tissues were composed of proliferating epithelial cells with a high nuclear/cytoplasmic ratio, and resembled the features of noninvasive tubular adenocarcinoma (Figure 2B). They exhibited tubular and cystic structures of varying sizes, and the large cystic structures contained coagulated red blood cells in the lumen (Figure 2B). The tumors rarely invaded the surrounding stroma. In some cases, mammary gland hyperplasia and adenoma were observed between the normal mammary gland and carcinoma tissues (Figure 2, C-G), indicating a possible sequential change from the normal to the malignant phenotypes. No distant metastases were found in mice carrying the breast tumors.

\section{Proliferating Breast Tumor Cells in Nrk Mutant Mice Express ER $\alpha$}

The onset and/or growth of breast tumor are partly regulated by estrogen, ${ }^{7}$ and the ER expression is a hallmark of adenoma and low-grade carcinoma of the mammary gland origin. ${ }^{11}$ We therefore examined the expression of ER $\alpha$ in the above-described $\mathrm{Nrk}^{-/-}$breast tumor specimen (Figure $2 \mathrm{C}$ ) that contained mammary gland hyperplasia and adenoma. As expected, a significant proportion of mammary epithelial cells in both normal (Figure 3A) and hyperplastic (Figure 3B) tissues were stained positive for ER $\alpha$. The proportion of ER $\alpha$-expressing cells increased in the adenoma tissue (Figure 3C), and carcinoma cells continued to express the receptor (Figure 3D). Counting the number of ER $\alpha$-positive cells in the carcinoma tissue revealed that $47.1 \% \pm 5.8 \%$ of the carcinoma cells express ER $\alpha$ (Figure 3E). Immunohistochemical staining of a serial section for Ki-67, followed by cell counting, showed that
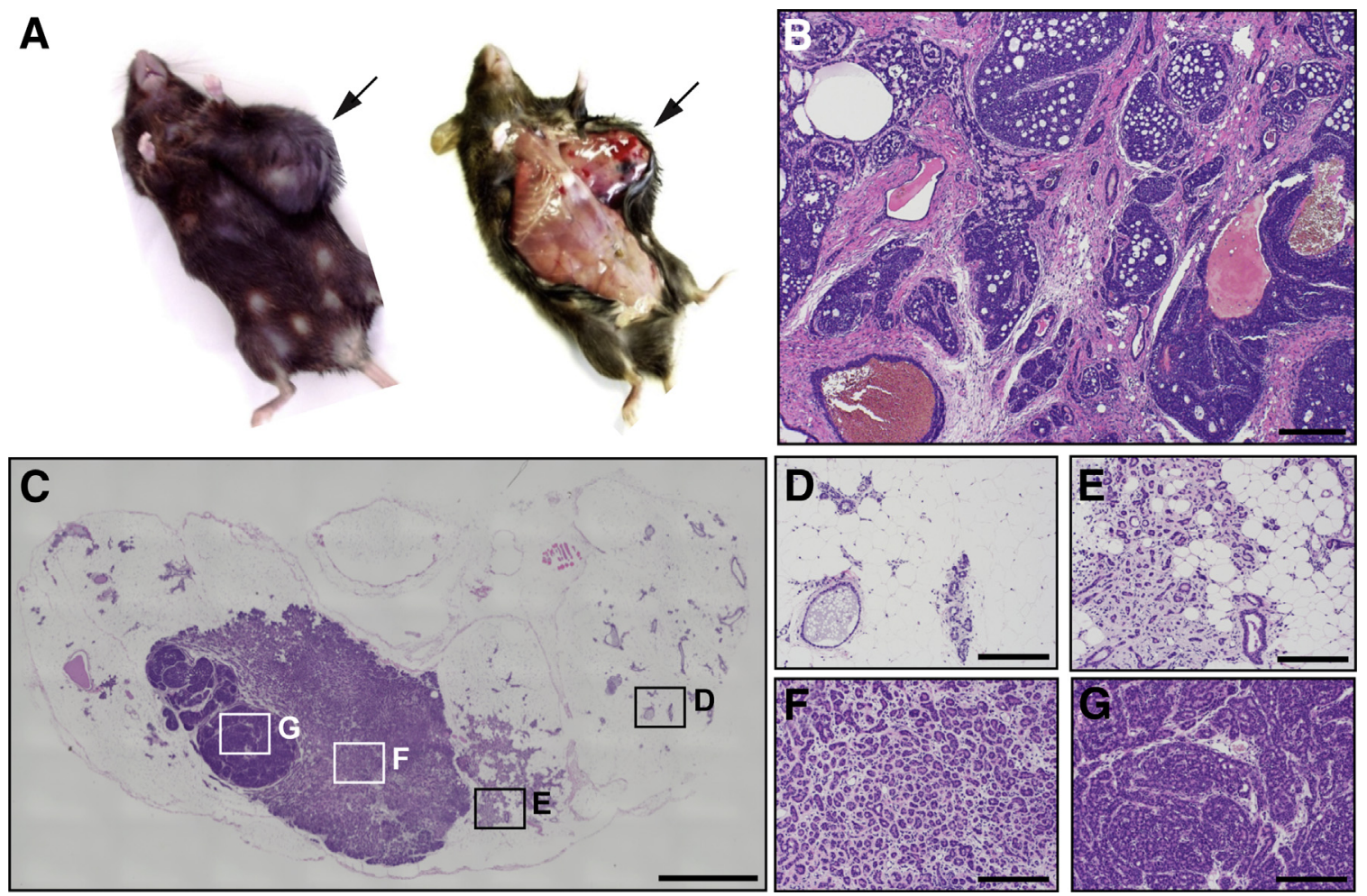

Figure 2 Macroscopic and histopathological features of breast tumor in $\mathrm{Nrk}^{-/-}$mice. A: Representative images of a $\mathrm{Nrk}^{-1-}$ mouse developing a large breast tumor (arrows) in the left anterior mammary gland. B: Hematoxylin and eosin (H\&E) staining of a breast tumor, composed of proliferating epithelial cells forming varying sizes of tubular and cystic structures, in a $\mathrm{Nrk}^{-1-}$ mouse. C: Mammary hyperplasia (box E) and adenoma (box F) were occasionally observed by H\&E staining between the normal (box $\mathbf{D}$ ) and carcinoma (box G) tissues in the mammary tumor of $\mathrm{Nrk}^{-/-}$mice. D-G: The boxed areas in $\mathbf{C}$ are shown in a higher magnification. Scale bars: $200 \mu \mathrm{m}(\mathbf{B}$ and $\mathbf{D}-\mathbf{G}) ; 2 \mathrm{~mm}(\mathbf{C})$. 

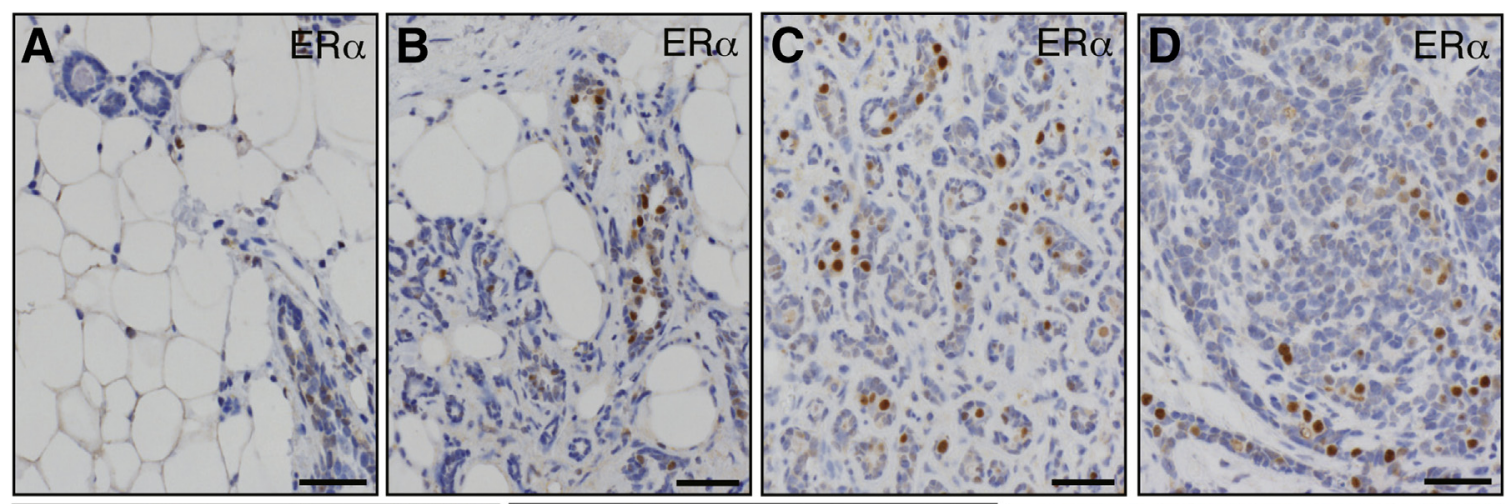

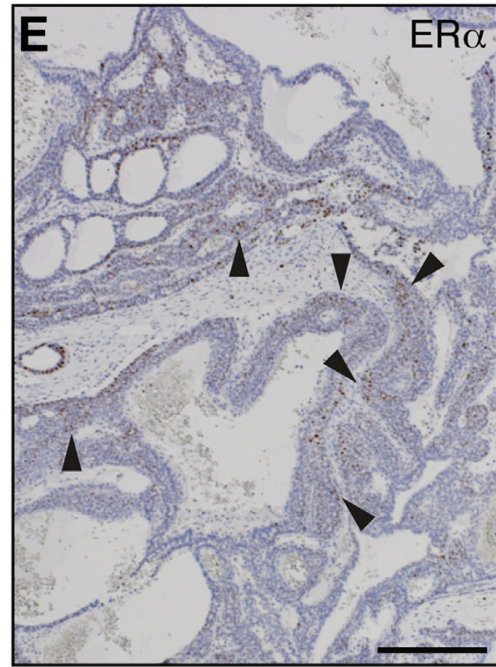

$47.1 \% \pm 5.8 \%$
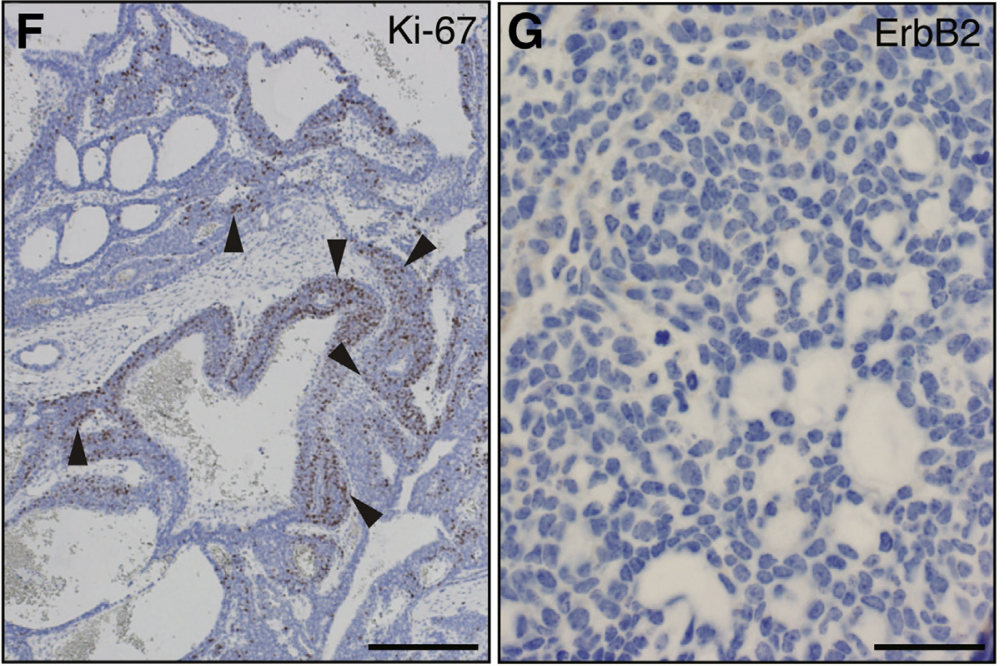

$26.3 \% \pm 2.0 \%$

Figure 3 Estrogen receptor (ER) $\alpha$ expression in breast tumor in $\mathrm{Nrk}^{-/-}$mice. Tissue sections prepared from the breast tumors in $\mathrm{Nrk}^{-/-}$mice were stained with specific antibodies recognizing $\operatorname{ER} \alpha(\mathbf{A}-\mathbf{E}), \mathrm{Ki}-67(\mathbf{F})$, or ErbB2 (G). Representative images were taken from normal (A), hyperplasia (B), adenoma (C), and carcinoma (D-G) tissues. $\mathbf{E}$ and $\mathbf{F}$ are serial sections. Arrowheads indicate representative regions showing coexpression of ER $\alpha$ and Ki-67. The proportion of ER $\alpha$ - and Ki-67-positive cells was calculated by counting cells in five sections (2000 cells per section) for each staining, and shown below each panel. All of the sections were counterstained with hematoxylin. Scale bars: $50 \mu \mathrm{m}$ (A-D and G); $200 \mu \mathrm{m}$ (E and F).

$26.3 \% \pm 2.0 \%$ of the carcinoma cells were positive for this proliferating cell marker (Figure 3F). Some of the ER $\alpha$ positive carcinoma cells coexpressed Ki-67 (Figure 3, E and F). On the other hand, the carcinoma cells did not express ErbB2/HER2 (Figure 3G).

\section{Nrk Mutant Mammary Gland Contains ER $\alpha$-Positive Hyperplastic Foci at Late Gestation}

We next examined whether the Nrk mutation affects the development of the mammary gland and its physiological hyperplasia during pregnancy in female mice. Hematoxylin and eosin staining and immunohistochemical staining of $\mathrm{ER} \alpha$, followed by measuring of the cell area and counting of the cell number, showed that there was no significant difference in the levels of mammary epithelial cell proliferation (Figure 4, A and B) or ER $\alpha$ expression (Figure 4, E and F) in the mammary gland between WT and $\mathrm{Nrk}^{-/-}$ mice that had not experienced pregnancy/parturition. A similar degree of mammary hyperplasia was also observed in pregnant WT (Figure 4C) and $\mathrm{Nrk}^{-/-}$(Figure 4D) mice at $17.5 \mathrm{dpc}$. In most mammary glands, the ER $\alpha$ expression in the physiologically proliferated mammary epithelial cells was down-regulated at this late period of gestation, and there was no difference in the number of ER $\alpha$-expressing cells between WT and $\mathrm{Nrk}^{-1-}$ mice (Figure 4, G and H). However, a certain proportion of the mammary glands in $\mathrm{Nrk}^{--}$, but not WT, mice ( 2 of 12 specimens) contained hyperplastic foci that consistently expressed $\mathrm{ER} \alpha$, even at 17.5 dpc (Figure 4, I and J). These results raised the possibility that in Nrk mutant mice, estrogen-dependent hyperproliferation of mammary epithelial cells already occurs during pregnancy and some of these lesions develop into the breast tumor subsequently.

\section{Nrk Expression Is Induced in the Mammary Gland at Late Gestation}

We then investigated how Nrk deficiency leads to the development of breast tumor. Previous studies have shown 


\section{Non-pregnant}

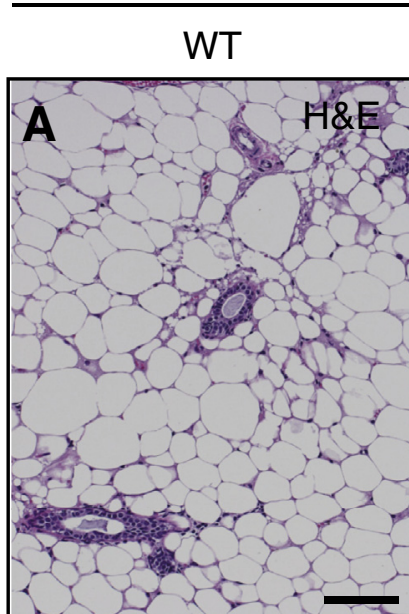

$3.2 \% \pm 0.8 \%$

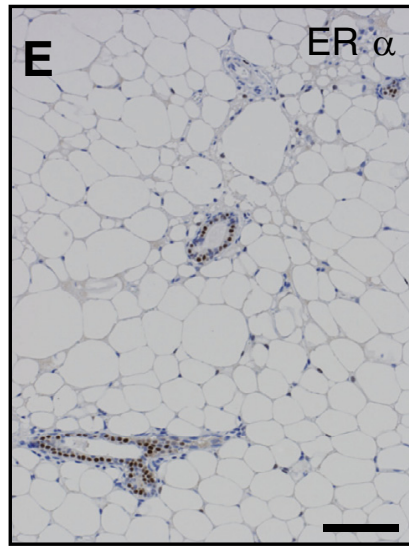

$41.7 \% \pm 6.1 \%$

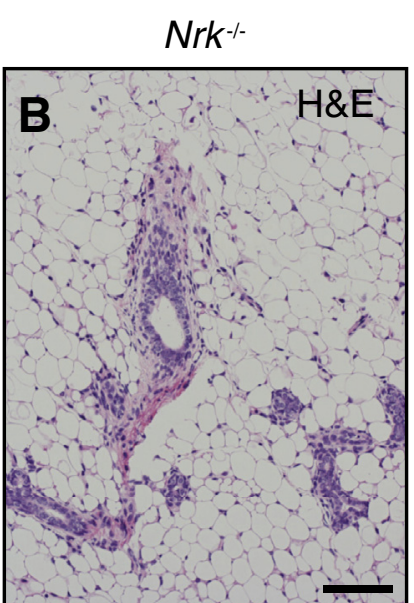

$3.4 \% \pm 1.8 \%$

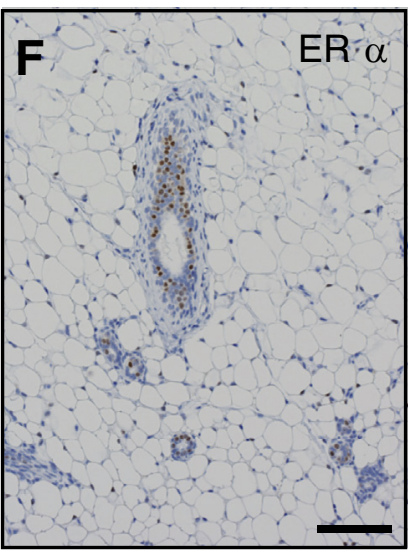

$35.8 \% \pm 8.6 \%$

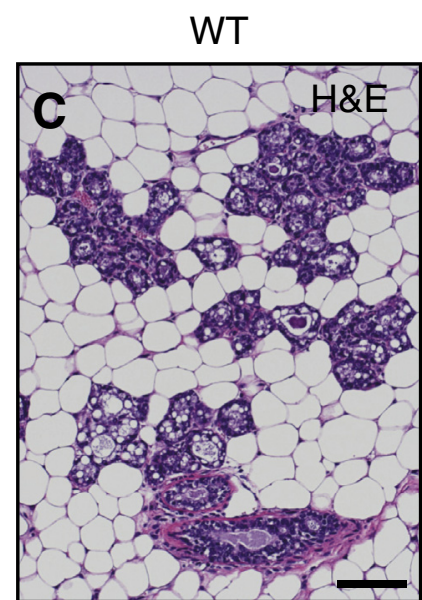

$40.0 \% \pm 6.6 \%$

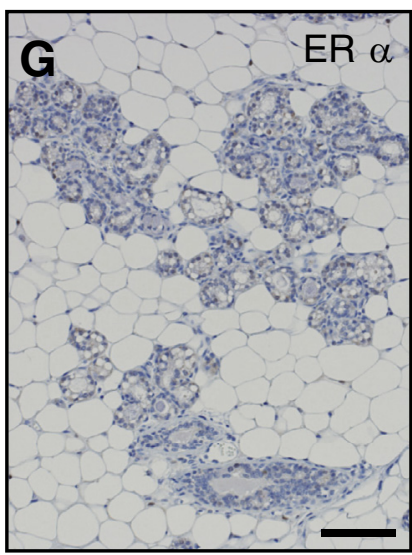

$38.4 \% \pm 8.3 \%$
$17.5 \mathrm{dpc}$

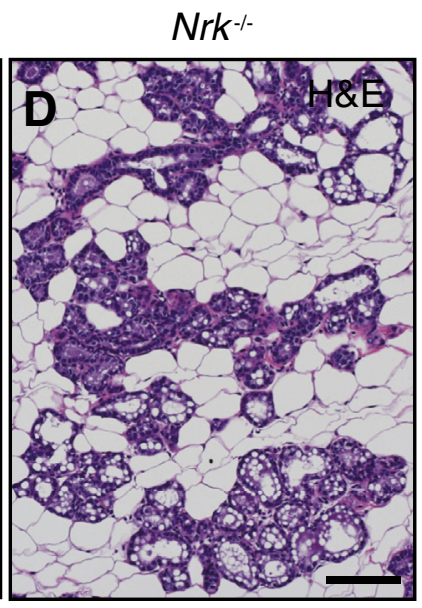

$37.5 \% \pm 5.1 \%$

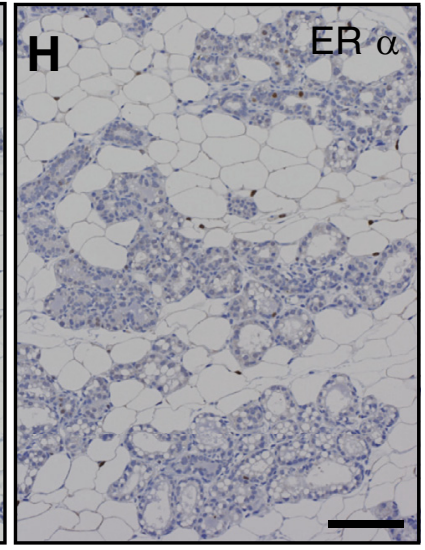

$35.2 \% \pm 9.5 \%$

$\mathrm{Nrk}^{-1-}$ at $17.5 \mathrm{dpc}$

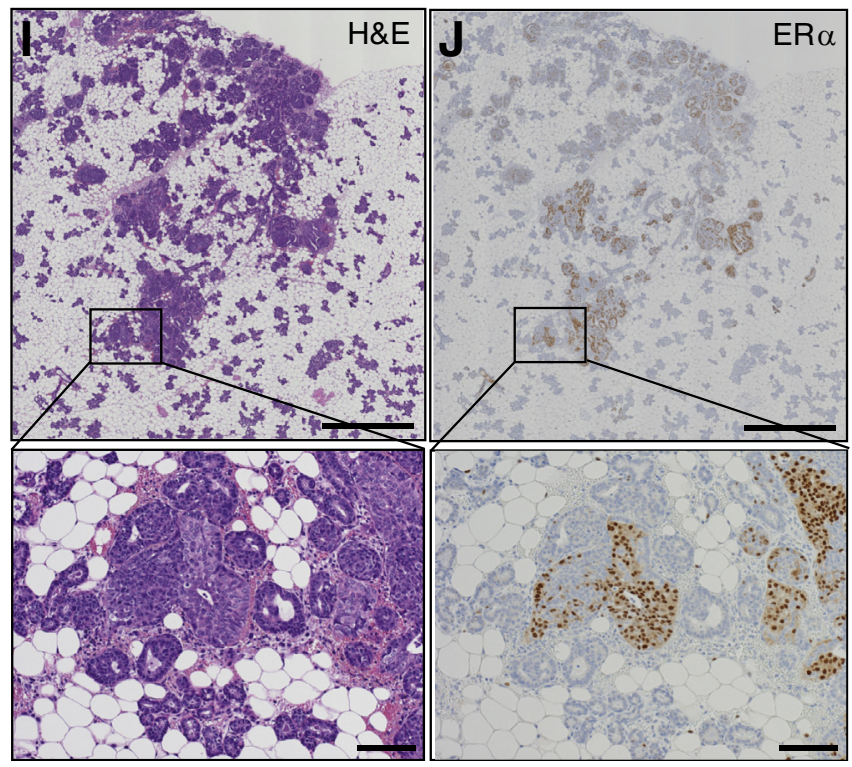

Figure 4 Mammary epithelial cell proliferation and estrogen receptor (ER) $\alpha$ expression in the mammary gland of non-pregnant and pregnant wild-type (WT) and $\mathrm{Nrk}^{-/-}$mice. Mammary glands were dissected from WT (A, C, E, and G) and $\mathrm{Nrk}^{-/-}$(B, D, F, H, I, and $\mathbf{J}$ ) female mice harboring no breast tumor. The tissues were from mice without a history of pregnancy/parturition (A, B, E, and $\mathbf{F}$ ) or pregnant mice at 17.5 days postcoitum (dpc; C, D, G, H, I, and J). Serial sections were examined by hematoxylin and eosin (H\&E) staining (A-D and I) and immunohistochemical staining with anti$E R \alpha$ antibody $(\mathbf{E}-\mathbf{H}$ and $\mathbf{J})$. The boxed areas in $\mathbf{I}$ and $\mathbf{J}$ are shown below in a higher magnification. The relative areas (\%) of mammary epithelial cells (A-D) and the proportion (\%) of ER $\alpha$-expressing mammary epithelial cells $(\mathbf{E}-\mathbf{H})$, evaluated in randomly chosen 10 individual visual fields for each specimen, are indicated below panels A-H. Scale bars: $100 \mu \mathrm{m}$ (A-H and bottom panels in I and J); $1 \mathrm{~mm}$ (top panels in $\mathbf{I}$ and $\mathbf{J}$ ). 

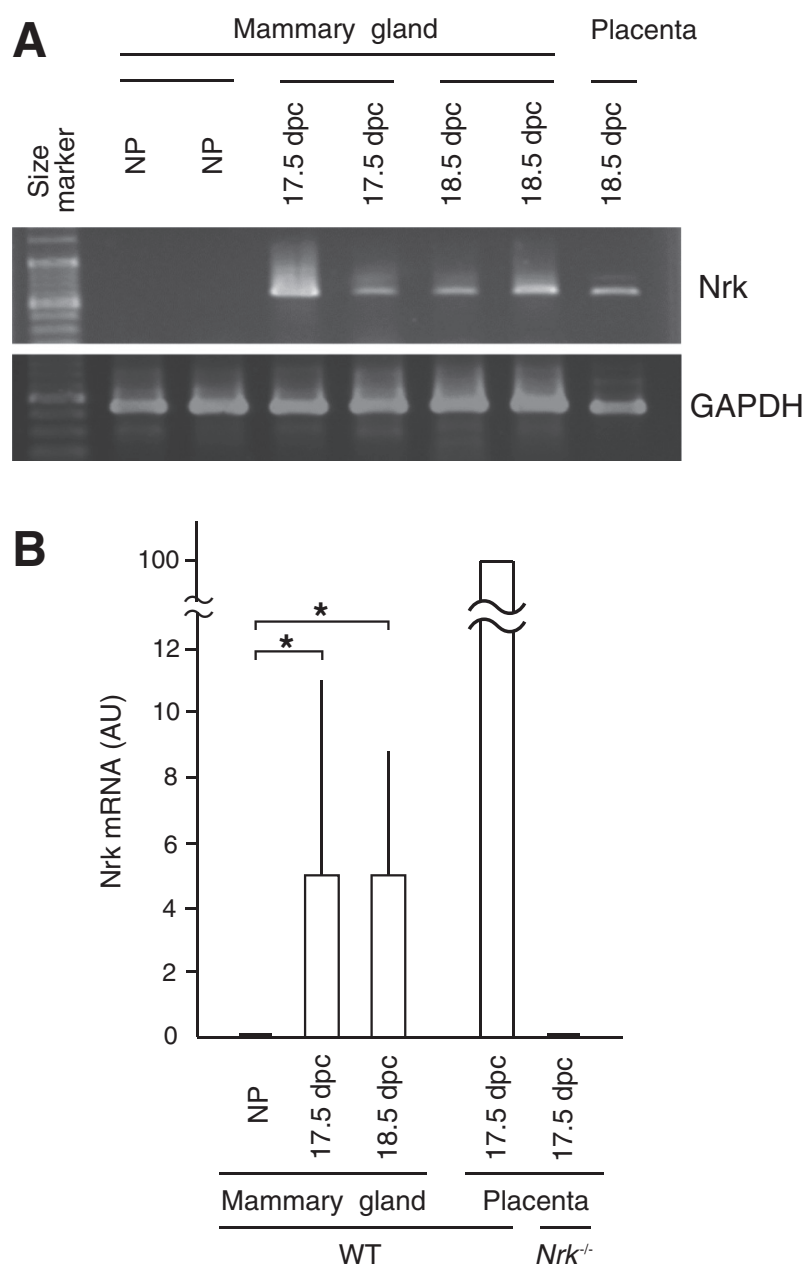

Figure 5 Induced Nrk mRNA expression in the mammary gland in female mice at late gestation. Expression of Nrk mRNA was examined by RT-PCR (A) and real-time RT-PCR (B) using total RNAs from the mammary glands of wild-type (WT) female mice without a history of pregnancy/ parturition [non-pregnant (NP)] or at late gestation [17.5 and 18.5 days postcoitum (dpc)] as templates. Total RNAs from WT and $\mathrm{Nrk}^{-/-}$placentas (17.5 or $18.5 \mathrm{dpc}$ ) were used as positive and negative controls, respectively, for the detection of Nrk mRNA. Glyceraldehyde 3-phosphate dehydrogenase (GAPDH) CDNA was amplified as an internal control. $n=2$ for each group (A); $n=3$ for each group (B). ${ }^{*} P<0.05$. AU, arbitrary unit; GAPDH, glyceraldehyde-3-phosphate dehydrogenase.

that Nrk mRNA is expressed restrictedly in the embryo and placenta but not in any adult tissues examined in mice. ${ }^{18-20}$ However, Nrk expression in the mammary gland has not been examined so far. We therefore isolated total RNA from the tissue and performed the RT-PCR (Figure 5A) and realtime RT-PCR (Figure 5B) analyses. Nrk cDNA was not amplified from the mammary gland of WT female mice that had experienced no pregnancy/parturition. In striking contrast, Nrk expression was remarkably up-regulated in the mammary gland in pregnant WT mice at 17.5 and $18.5 \mathrm{dpc}$. As positive and negative controls, respectively, Nrk cDNA was also amplified from total RNAs of WT but not $\mathrm{Nrk}^{-1-}$ placentas. These results suggested that the lack of Nrk expression in the mammary gland affects the proliferation of mammary epithelial cells at late gestation, leading to subsequent development of breast tumor.

\section{Nrk Does Not Regulate the Expression or Activity of $\mathrm{ER} \propto$ Directly}

To examine the possibility that Nrk regulates ER $\alpha$ expression and/or function directly, we performed several experiments. We first compared the level of ER $\alpha$ mRNA expression in pregnant WT and $\mathrm{Nrk}^{-1-}$ mammary glands using real-time RT-PCR analysis. Consistent with the results of immunohistochemical staining (Figure 4), there was no difference in the levels of ER $\alpha$ expression between WT and $\mathrm{Nrk}^{-1-}$ tissues either in the non-pregnant condition or at $17.5 \mathrm{dpc}$ (Figure 6A).

We next used the MCF-7 human breast cancer cells that express ER $\alpha$ and respond to estrogen, and examined the effect of Nrk overexpression on the expression and phosphorylation of the ER $\alpha$ protein. Immunoblotting of the lysates of MCF-7 cells overexpressing WT or kinase-dead (K54E) mutant of Nrk showed that the levels of endogenous ER $\alpha$ protein were unchanged by expression of WT or K54E mutant Nrk (Figure 6B). Phos-tag SDS-PAGE, which facilitates the separation of phosphorylated and non-phosphorylated protein species, ${ }^{27}$ of the same cell lysates followed by anti-ER $\alpha$ immunoblotting revealed no change in the electrophoretic pattern of ER $\alpha$ on Nrk overexpression, suggesting that Nrk also does not affect the phosphorylation state of $\mathrm{ER} \alpha$ (Figure 6B).

Finally, the effect of Nrk overexpression on ER $\alpha$ dependent target gene expression was examined using a luciferase reporter construct harboring three tandem EREs. Estrogen treatment of MCF-7 cells resulted in a significant increase in the ERE luciferase reporter expression in the absence of Nrk overexpression (Figure 6C). However, cotransfection of WT or K54E mutant Nrk did not alter the level of reporter expression (Figure 6C). Collectively, these results suggested that Nrk does not have a direct effect on the expression or transcriptional activity of ER $\alpha$.

\section{Blood Estrogen Levels Are Elevated in Nrk Mutant Pregnant Mice at Late Gestation}

We finally asked whether the estrogen levels are affected in the blood circulation of pregnant $N r k^{-1-}$ mice. We crossed $N r k^{-1-}$ and WT females ( $n=5$ for each group) to WT males. Blood was collected from the abdominal vena cava of the pregnant mice at $17.5 \mathrm{dpc}$, and the serum concentrations of estradiol and progesterone were measured using the liquid chromatography-tandem mass spectrometry analysis. We found that the serum estradiol levels were elevated in $\mathrm{Nrk}^{-1-}$ mice compared to WT mice (27.6 \pm 8.2 and $13.8 \pm 4.7 \mathrm{pg} / \mathrm{mL}$ for $\mathrm{Nrk}^{-1-}$ and WT, respectively; $P<0.05$ ) (Figure 7A). On the other hand, the progesterone levels were not significantly altered in the absence of $\mathrm{Nrk}$ 
A

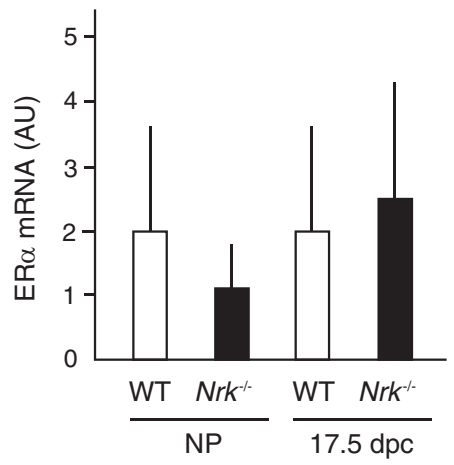

B

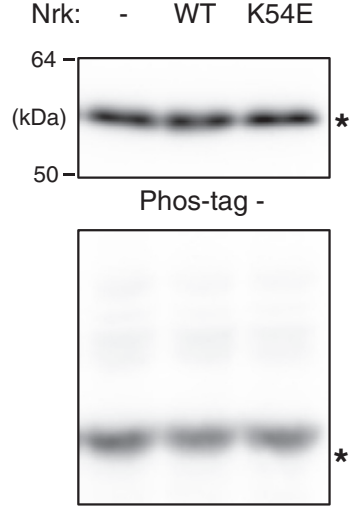

Phos-tag +
C

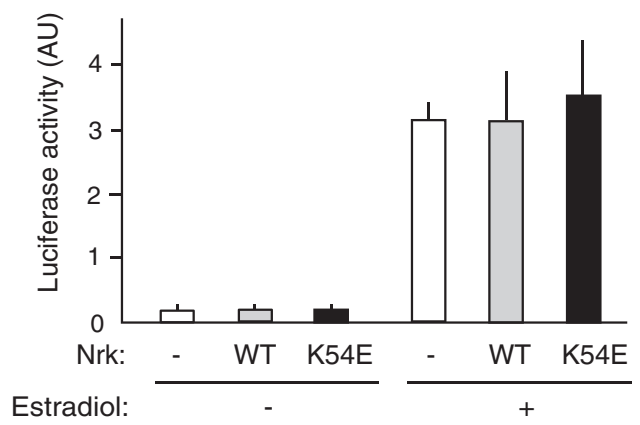

Figure 6 Effects of Nrk deletion and overexpression on estrogen receptor (ER) $\alpha$ expression and activity. A: Total RNA was extracted from the mammary glands of non-pregnant (NP) or pregnant [17.5 days postcoitum (dpc)] wild-type (WT) and $\mathrm{Nrk}^{-/}$mice. The amounts of ER $\alpha$ mRNA were quantified by realtime RT-PCR analysis. B: MCF-7 cells were transfected with an expression construct encoding WT or K54E mutant of Nrk. The lysates of the cells were subjected to SDS-PAGE in the absence (top panel) or presence (bottom panel) of Phos-tag, followed by immunoblotting with anti-ER $\alpha$ antibody. Asterisks indicate the position of ER $\alpha$. C: MCF-7 cells were cotransfected with the estrogen responsive element luciferase reporter construct and an expression construct encoding WT or K54E mutant of Nrk. Cells were treated with or without $10 \mathrm{nmol} / \mathrm{L}$ estradiol for 48 hours, and the luciferase activity was measured in the cell lysates. $n=3$ (A and $\mathbf{C})$. $\mathrm{AU}$, arbitrary unit.

$\left(35.4 \pm 14.6\right.$ and $41.3 \pm 5.2 \mathrm{ng} / \mathrm{mL}$ for $\mathrm{Nrk}^{-\prime-}$ and $\mathrm{WT}$, respectively; $P>0.05$ ) (Figure 7B).

In an attempt to identify the tissue(s) responsible for the elevated blood estrogen level in $\mathrm{Nr}^{-1-}$ mice, we examined the expression level of mRNA for CYP19A1, a key enzyme in estrogen synthesis, in the ovary and placenta by real-time RT-PCR analysis. We detected the CYP19A1 expression only in the ovary, but the expression levels were unchanged between the WT and $\mathrm{Nr}^{-/-}$tissues either in the nonpregnant condition or at $17.5 \mathrm{dpc}$ (Figure $7 \mathrm{C}$ ).

\section{Discussion}

In the present study, we demonstrate a frequent occurrence of breast tumor in Nrk mutant female mice that have experienced pregnancy/parturition. The tumors exhibited the histopathological features of noninvasive tubular adenocarcinoma with rare stromal invasion. Approximately a half of the carcinoma cells expressed ER $\alpha$ (Figure 3), and ER $\alpha$-positive mammary gland hyperplasia and adenoma were sometimes observed between the normal mammary
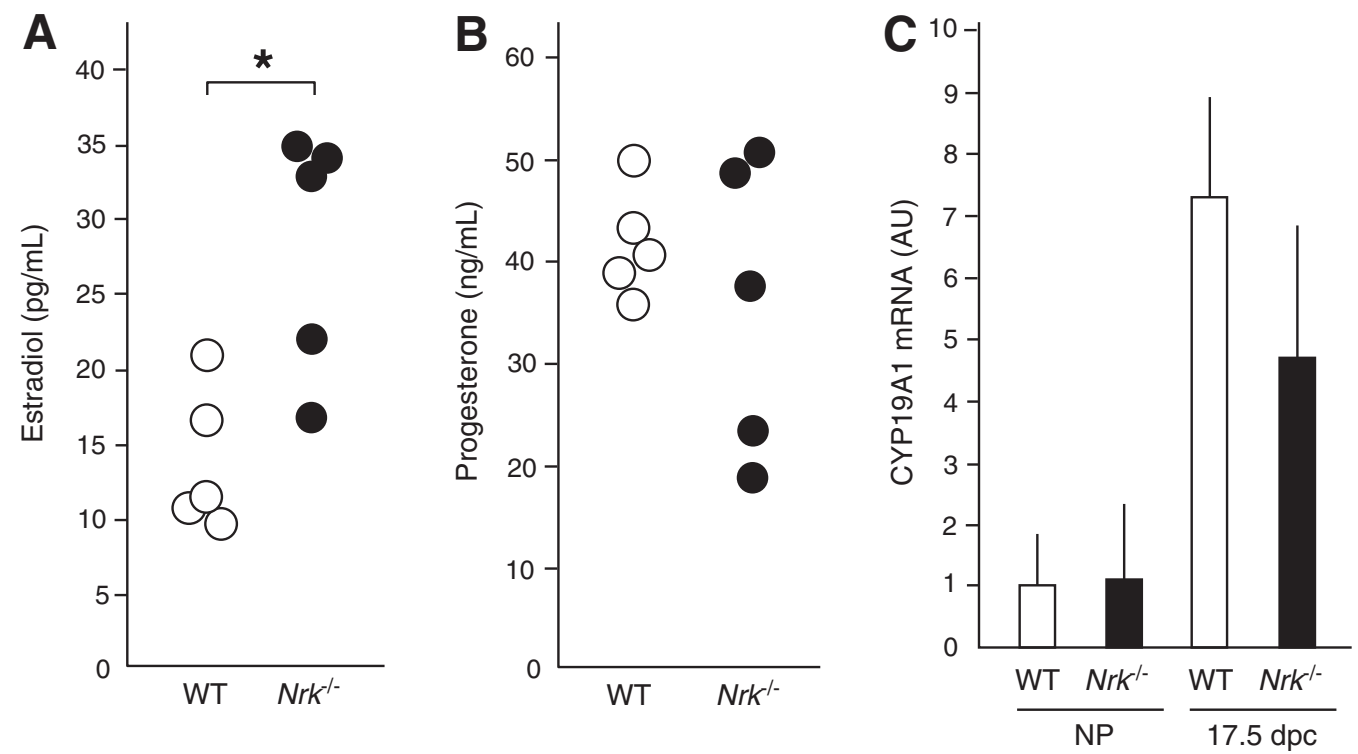

Figure 7 Levels of blood estrogen and CYP19A1 expression in the ovary in Nrk ${ }^{-1}$ mice. A and B: The serum concentrations of estradiol (A) and progesterone (B) were examined using liquid chromatography-tandem mass spectrometry in pregnant [17.5 days postcoitum (dpc)] wild-type (WT) and Nrk ${ }^{-/}$ mice that had been mated with WT male mice. C: Total RNA was extracted from the ovary of non-pregnant (NP) or pregnant (17.5 dpc) WT and Nrk ${ }^{-/-}$mice. The amounts of CYP19A1 mRNA were quantified by real-time RT-PCR analysis. $n=5$ for each group (A and $\mathbf{B}) ; n=3(\mathbf{C})$. ${ }^{\star} P<0.05$. AU, arbitrary unit. 
gland and carcinoma tissues (Figures 2 and 3). This may represent an estrogen-dependent sequential change from the normal to the malignant phenotypes, which resembles the multistep breast tumorigenesis in humans. ${ }^{28} \mathrm{~A}$ substantial proportion $(26.3 \%)$ of the carcinoma cells exhibited Ki-67 expression, whereas they were negative for ErbB2/HER2 (Figure 3). These results indicated that the breast tumors in the Nrk mutant mice are most likely to represent the luminal B (HER2-negative) subtype of human breast cancer. ${ }^{29}$ Further experiments, including extensive gene expression profiling using breast cancer classifier panels, are necessary to determine whether the Nrk mutant mice can serve as an appropriate model for a certain kind of human breast cancer.

When continuously mated with WT male mice, breast tumor developed in 9 of 10 (90\%) $\mathrm{Nrk}^{-/-}$mice and 5 of 10 $(50 \%) \mathrm{Nrk}^{+/-}$mice (Figure 1). This difference in the frequency of tumor development can clearly be explained as an effect of X-inactivation, a phenomenon in which one of the two X chromosomes in eutherian female mammals is transcriptionally inactivated, although incompletely, at early stages of embryogenesis. ${ }^{30,31}$ Given that the inactivation occurs randomly between the two $\mathrm{X}$ chromosomes, approximately $50 \%$ of the mammary epithelial cells in $\mathrm{Nrk}^{+/-}$mice are nearly $\mathrm{Nrk}$-null because the $\mathrm{X}$ chromosome harboring the WT Nrk allele is inactivated in $50 \%$ of the cells. Therefore, assuming that the breast tumor arises from Nrk-null cells, $\mathrm{Nrk}^{+/-}$mice have a $50 \%$ chance of breast tumor incidence compared to $\mathrm{Nrk}^{-1-}$ mice.

A certain proportion of the grossly normal mammary glands in pregnant $N r k$ mutant mice contained hyperplastic foci that consistently expressed ER $\alpha$ at $17.5 \mathrm{dpc}$ (Figure 4), a stage when ER $\alpha$ expression is down-regulated in physiologically proliferated WT mammary epithelial cells. ${ }^{32}$ More important, we found that the Nrk expression in the WT mammary gland, which was undetectable in female mice with no history of pregnancy/parturition, was induced during this late period of gestation (Figure 5). These findings implicate Nrk in preventing the overgrowth of physiologically proliferated mammary epithelial cells at late gestation. However, the level of ER $\alpha$ mRNA expression was not elevated in the $\mathrm{Nrk}^{-1-}$ mammary gland compared to the WT tissue (Figure 6). In vitro experiments using MCF-7 cells overexpressing Nrk also suggested that the Nrk kinase does not regulate the levels of expression, phosphorylation, or transcription factor activity of ER $\alpha$ (Figure 6). Therefore, Nrk may not directly down-regulate the ER $\alpha$ activity. From not only cell biological but also clinical viewpoints, it is essential to elucidate the mechanisms by which Nrk suppresses the overproliferation of mammary epithelial cells. A previous study in human cultured cells showed that Nrk mediates the apoptotic signaling triggered by tumor necrosis factor- $\alpha .^{22}$ Another possibility, therefore, is that Nrk prevents the uncontrolled hyperplasia of the mammary gland by inducing the apoptosis of excessively proliferating ER $\alpha$ positive mammary epithelial cells at late gestation.
The increase in the blood estrogen levels at $17.5 \mathrm{dpc}$ in $\mathrm{Nrk}^{-1-}$ pregnant mice was rather modest; the hormone levels were at most twice higher than in WT mice (Figure 7). However, several epidemiological studies indicated that the mildly elevated blood estrogen level accounts for the increased risk of breast cancer occurrence. ${ }^{5}$ The varying levels of blood estrogen in the pregnant $\mathrm{Nrk}^{-1-}$ mice (Figure 7) and the presence of ER $\alpha$-positive hyperplastic foci in a certain proportion of the $\mathrm{Nrk}^{-1-}$ mammary glands (Figure 4) may suggest the presence of a threshold for the initiation of breast tumor development. Namely, the breast tumor in Nrk mutant mice may be triggered when multiple factors, such as ER $\alpha$ expression/activity in mammary epithelial cells and estrogen concentration in the blood, reach certain critical levels, and the chance for this may increase as the number of pregnancy/parturition increases. After the onset of the breast tumor (7 months of age), however, the number of mice carrying the tumor increased proportionally, rather than acceleratingly or exponentially, to their age (Figure 1). These results suggest that the trigger factor for the tumorigenesis does not accumulate through repetitive pregnancy/parturition but is reset after each pregnancy/parturition. As reported previously, ${ }^{33}$ the CYP19A1 expression was lower than the detectable level in the placenta in both WT and $\mathrm{Nrk}^{-/-}$mice, and although it was highly expressed in the ovary, the levels were unchanged in the absence of Nrk. Therefore, the tissue responsible for the elevated blood estrogen levels in $\mathrm{Nrk}^{-1-}$ mice at late gestation remains to be determined.

The present study sheds light on the pathogenesis of estrogen-induced breast tumor. Future studies are necessary to precisely understand how Nrk suppresses the tumorigenesis of mammary epithelial cells. The COSMIC database summarizes 16 somatic Nrk mutations identified in approximately 1400 breast cancer genomes (Catalog of Somatic Mutations in Cancer, http://cancer.sanger.ac.uk/ cosmic, last accessed June 7, 2016). However, they were all found at different nucleotide positions, and no common mutation has been linked to breast cancer development. Because Nrk mutations possibly increase the susceptibility to breast tumor also in humans, it is important to continuously examine the presence of $\mathrm{Nrk}$ mutations in patients with breast tumor. A combination of these studies will eventually contribute to the development of novel preventive and therapeutic means for breast cancer.

\section{Acknowledgments}

We thank the staff in the Center for Biological Resources and Informatics (Tokyo Institute of Technology) for maintenance of the mouse colony and the Support Center for Medical Research and Education (Tokai University School of Medicine) for technical support in histological experiments. 


\section{References}

1. Campeau PM, Foulkes WD, Tischkowitz MD: Hereditary breast cancer: new genetic developments, new therapeutic avenues. Hum Genet 2008, 124:31-42

2. Zhang L, Long X: Association of BRCA1 promoter methylation with sporadic breast cancers: evidence from 40 studies. Sci Rep 2015, 5: 17869

3. Hunter DJ, Willett WC: Diet, body size, and breast cancer. Epidemiol Rev 1993, 15:110-132

4. Huang Z, Hankinson SE, Colditz GA, Stampfer MJ, Hunter DJ, Manson JE, Hennekens CH, Rosner B, Speizer FE, Willett WC: Dual effects of weight and weight gain on breast cancer. JAMA 1997, 278: 1407-1411

5. Key TJ, Appleby PN, Reeves GK, Dorgan JF, Stanczyk FZ, Stephenson HE Jr, et al: Body mass index, serum sex hormones, and breast cancer risk in postmenopausal women. J Natl Cancer Inst 2003, 95:1218-1226

6. Kaaks R, Berrino F, Key T, Rinaldi S, Dossus L, Biessy C, et al: Serum sex steroids in premenopausal women and breast cancer risk within the European Prospective Investigation into Cancer and Nutrition (EPIC). J Natl Cancer Inst 2005, 97:755-765

7. Bulun SE, Chen D, Moy I, Brooks DC, Zhao H: Aromatase, breast cancer and obesity: a complex interaction. Trends Endocrinol Metab 2012, 23:83-89

8. Kelsey JL, Gammon MD, John EM: Reproductive factors and breast cancer. Epidemiol Rev 1993, 15:36-47

9. Collaborative Group on Hormonal Factors in Breast Cancer: Breast cancer and hormonal contraceptives: collaborative reanalysis of individual data on 53297 women with breast cancer and 100239 women without breast cancer from 54 epidemiological studies. Lancet 1996, 347:1713-1727

10. The Women's Health Initiative Steering Committee: Effects of conjugated equine estrogen in postmenopausal women with hysterectomy: the women's health initiative randomized controlled trial. JAMA 2004, 291:1701-1712

11. Lumachi F, Brunello A, Maruzzo M, Basso U, Basso SM: Treatment of estrogen receptor-positive breast cancer. Curr Med Chem 2013, 20: 596-604

12. Early Breast Cancer Trialists' Collaborative Group: Effects of chemotherapy and hormonal therapy for early breast cancer on recurrence and 15-year survival: an overview of the randomized trials. Lancet 2005, 365:1687-1717

13. Forbes JF, Cuzick J, Buzdar A, Howell A, Tobias JS, Baum M: Effect of anastrozole and tamoxifen as adjuvant treatment for early-stage breast cancer: 100-month analysis of the ATAC trial. Lancet Oncol 2008, 9: $45-53$

14. Abbassi-Ghanavati M, Greer LG, Cunningham FG: Pregnancy and laboratory studies: a reference table for clinicians. Obstet Gynecol 2009, 114:1326-1331

15. Joshi PA, Di Grappa MA, Khokha R: Active allies: hormones, stem cells and the niche in adult mammopoiesis. Trends Endocrinol Metab 2012, 23:299-309

16. Lambe M, Hsieh C, Trichopoulos D, Ekbom A, Pavia M, Adami HO: Transient increase in the risk of breast cancer after giving birth. N Engl J Med 1994, 331:5-9

17. Guzman RC, Yang J, Rajkumar L, Thordarson G, Chen X, Nandi S: Hormonal prevention of breast cancer: mimicking the protective effect of pregnancy. Proc Natl Acad Sci U S A 1999, 96:2520-2525
18. Nakano K, Yamauchi J, Nakagawa K, Itoh H, Kitamura N: NESK, a member of the germinal center kinase family that activates the c-Jun $\mathrm{N}$ terminal kinase pathway and is expressed during the late stages of embryogenesis. J Biol Chem 2000, 275:20533-20539

19. Kanai-Azuma M, Kanai Y, Okamoto M, Hayashi Y, Yonekawa H, Yazaki K: Nrk: a murine X-linked NIK (Nck-interacting kinase)related kinase gene expressed in skeletal muscle. Mech Dev 1999, 89:155-159

20. Denda K, Nakao-Wakabayashi K, Okamoto N, Kitamura N, Ryu JY, Tagawa Y, Ichisaka T, Yamanaka S, Komada M: Nrk, an X-linked protein kinase in the germinal center kinase family, is required for placental development and fetoplacental induction of labor. J Biol Chem 2011, 286:28802-28810

21. Nakano K, Kanai-Azuma M, Kanai Y, Moriyama K, Yazaki K, Hayashi Y, Kitamura N: Cofilin phosphorylation and actin polymerization by NRK/NESK, a member of the germinal center kinase family. Exp Cell Res 2003, 287:219-227

22. Kakinuma H, Inomata H, Kitamura N: Enhanced JNK activation by NESK without kinase activity upon caspase-mediated cleavage during apoptosis. Cell Signal 2005, 17:1439-1448

23. Higashi K, Inagaki Y, Suzuki N, Mitsui S, Mauviel A, Kaneko H, Nakatsuka I: Y-box binding protein YB-1 mediates transcriptional repression of human $\alpha 2$ (I) collagen gene expression by interferon- $\gamma$. J Biol Chem 2003, 278:5156-5162

24. Hall JM, McDonnell DP: The estrogen receptor beta-isoform (ER $\beta$ ) of the human estrogen receptor modulates ER $\alpha$ transcriptional activity and is a key regulator of the cellular response to estrogens and antiestrogens. Endocrinology 1999, 140:5566-5578

25. Kutsukake N, Ikeda K, Honma S, Teramoto M, Mori Y, Hayasaka I Yamamoto R, Ishida T, Yoshikawa Y, Hasegawa T: Validation of salivary cortisol and testosterone assays in chimpanzees by liquid chromatography-tandem mass spectrometry. Am J Primatol 2009, 71: 696-706

26. Arai S, Miyashiro Y, Shibata Y, Kashiwagi B, Tomaru Y, Kobayashi M, Watanabe Y, Honma S, Suzuki K: New quantification method for estradiol in the prostatic tissues of benign prostatic hyperplasia using liquid chromatography-tandem mass spectrometry. Steroids 2010, 75:13-19

27. Kinoshita E, Kinoshita-Kikuta E, Koike T: Separation and detection of large phosphoproteins using Phos-tag SDS-PAGE. Nat Protoc 2009, 1: $1513-1521$

28. Tsubura A, Yoshizawa K, Uehara N, Yuri T, Matsuoka Y: Multistep mouse mammary tumorigenesis through preneoplasia to neoplasia and acquisition of metastatic potential. Med Mol Morphol 2007, 40: $9-17$

29. Pfefferle AD, Herschkowitz JI, Usary J, Harrell JC, Spike BT, Adams JR, Torres-Arzayus MI, Brown M, Egan SE, Wahl GM, Rosen JM, Perou CM: Transcriptomic classification of genetically engineered mouse models of breast cancer identifies human subtype counterparts. Genome Biol 2013, 14:R125

30. Briggs SF, Reijo Pera RA: X chromosome inactivation: recent advances and a look forward. Curr Opin Genet Dev 2014, 28:78-82

31. Galupa R, Heard E: X-chromosome inactivation: new insights into cis and trans regulation. Curr Opin Genet Dev 2015, 31:57-66

32. Saji S, Jensen EV, Nilsson S, Rylander T, Warner M, Gustafsson JA: Estrogen receptors $\alpha$ and $\beta$ in the rodent mammary gland. Proc Natl Acad Sci U S A 2000, 97:337-342

33. Simpson ER: Sources of estrogen and their importance. J Steroid Biochem Mol Biol 2003, 86:225-230 